\title{
Applications of the generalised Dirichlet integral inequality to the Neumann problem with fast-growing continuous data
}

\author{
Wei Li and Muhammad Aslam Zaprawa2*
}

\section{"Correspondence:}

m.zaprawa@outlook.com

${ }^{2}$ Department of Mathematics and

Computer Science, Faculty of

Science, Alexandria University,

Alexandria, 21511, Egypt

Full list of author information is

available at the end of the article

\begin{abstract}
By using the generalised Dirichlet integral inequality with continuous functions on the boundary of the upper half-space, we prove new types of solutions for the Neumann problem with fast-growing continuous data on the boundary. Given any harmonic function with its negative part satisfying similarly fast-growing conditions, we obtain weaker boundary integral condition.
\end{abstract}

Keywords: Neumann problem; Neumann integral; upper half-space

\section{Introduction}

Let $\mathbf{R}^{n}$ denote the $n$-dimensional Euclidean space, where $n \geq 3$. We denote two points $L$ and $N$ in $\mathbf{R}^{n}$ by $L=\left(x^{\prime}, x_{n}\right)$ and $N=\left(y^{\prime}, y_{n}\right)$, respectively, where $x^{\prime}=\left(x_{1}, x_{2}, \ldots, x_{n-1}\right), y^{\prime}=$ $\left(y_{1}, y_{2}, \ldots, y_{n-1}\right), x_{n} \in \mathbf{R}$ and $y_{n} \in \mathbf{R}$. The Euclidean distance of them is denoted by $|L-N|$. Let $E$ be a subset of $\mathbf{R}^{n}$, we denote the boundary and closure of it by $\partial E$ and $\bar{E}$, respectively.

The set

$$
\left\{L=\left(x^{\prime}, x_{n}\right) \in \mathbf{R}^{n} ; x_{n}>0\right\}
$$

is denoted by $\mathcal{T}_{n}$, which is called the upper half-space. Let $F$ be a subset of $\mathbf{R}_{+} \cup\{0\}$. Then two sets

$$
\left\{L=\left(x^{\prime}, x_{n}\right) \in \mathcal{T}_{n} ;|L| \in F\right\} \quad \text { and } \quad\left\{N=\left(y^{\prime}, 0\right) \in \partial \mathcal{T}_{n} ;|N| \in F\right\}
$$

are denoted by $\mathcal{T}_{n} E$ and $\partial \mathcal{T}_{n} E$, respectively.

Let $B_{n}(r)$ denote the open ball with center at the origin and radius $r$, where $r>0$. By $S_{n}(r)$ we denote $\mathcal{T}_{n} \cap \partial B_{n}(r)$. When $g$ is a function defined by $\sigma_{n}(r)=\mathcal{T}_{n} \cap B_{n}(r)$, the mean of $g$ is defined by

$$
\mathrm{M}(g)(r)=\frac{2 s_{n}}{r^{n-1}} \int_{\sigma_{n}(r)} g(L) d \sigma_{L}
$$

where $s_{n}$ is the surface area of $B_{n}(1)$ and $d \sigma_{L}$ is the surface element on $B_{n}(r)$ at $L \in \sigma_{n}(r)$.

(C) 2016 Li and Zaprawa. This article is distributed under the terms of the Creative Commons Attribution 4.0 International License (http://creativecommons.org/licenses/by/4.0/), which permits unrestricted use, distribution, and reproduction in any medium, provided you give appropriate credit to the original author(s) and the source, provide a link to the Creative Commons license, and indicate if changes were made. 
Let $h(L)$ be a function on $\mathcal{T}_{n}$. In this paper we denote $h^{+}=\max \{h, 0\}, h^{-}=-\min \{h, 0\}$ and $[c]$ is the integer part of $c$, where $c \in \mathbf{R}$. Let $\partial / \partial n$ denote differentiation along the inward normal into $\mathcal{T}_{n}$. We use the Lebesgue measure $d L=d x^{\prime} d x_{n}$, where $d x^{\prime}=d x_{1} \cdots d x_{n-1}$.

Let $f$ be a continuous function on $\partial \mathcal{T}_{n}$. If $h$ is a harmonic function on $\mathcal{T}_{n}$ and

$$
\lim _{L \rightarrow N \in \partial \mathcal{T}_{n}, L \in \mathcal{T}_{n}(\Omega)} \frac{\partial h(L)}{\partial x_{n}}=f(N),
$$

then we say that $h$ is a solution of the Neumann problem on $\mathcal{T}_{n}$ with respect to $f$.

The uniqueness and the existence of solutions of the Neumann problem on $\mathcal{T}_{n}$ with a continuous function on $\partial \mathcal{T}_{n}$ were given by $\mathrm{Su}$ (see $\left.[1,2]\right)$.

Theorem A (see [3], Theorem 1) Let $f(N)\left(N=\left(y^{\prime}, 0\right)\right)$ be a function continuous on $\partial \mathcal{T}_{n}$ such that

$$
\int_{\partial \mathcal{T}_{n}} \mid f\left(y^{\prime}\right)\left(|1+| y^{\prime} \mid\right)^{2-n} d y^{\prime}<+\infty
$$

Then the Neumann integral

$$
\mathbb{H}_{0, n}[f](L)=-\rho_{n} \int_{\partial \mathcal{T}_{n}} f(N)|L-N|^{2-n} d N
$$

is a solution of the Neumann problem on $\mathcal{T}_{n}$ with respect to $f$ satisfying

$$
\mathrm{M}\left(\mathbb{H}_{0, n}[f]\right)(r)=O(1)
$$

as $r \rightarrow+\infty$, where $\rho_{n}=2\left\{(n-2) s_{n}\right\}^{-1}$.

Theorem B (see [3], Theorem 3) Let $k$ be a positive integer, $f$ be a continuous function on $\partial \mathcal{T}_{n}$ such that (1.1) holds and $h(L)$ be a solution of the Neumann problem on $\mathcal{T}_{n}$ with respect tof satisfying

$$
\mathrm{M}\left(h^{+}\right)(r)=o\left(r^{k}\right)
$$

as $r \rightarrow+\infty$. Then

$$
h(L)=\mathbb{H}_{0, n}(f)(L)+ \begin{cases}d & \text { when } k=1, \\ \Pi\left(x^{\prime}\right)+\sum_{j=1}^{\left[\frac{k}{2}\right]} \frac{(-1)^{j}}{(2 j) !} x_{n}^{2 j} \Delta^{j} \Pi\left(x^{\prime}\right) & \text { when } k \geq 2,\end{cases}
$$

for any $L=\left(x^{\prime}, x_{n}\right)$, where $d$ is a constant, $\Pi\left(x^{\prime}\right)$ is a polynomial of degree less than $k$ on $\partial \mathcal{T}_{n}$ and

$$
\Delta^{j}=\left(\frac{\partial^{2}}{\partial x_{1}^{2}}+\frac{\partial^{2}}{\partial x_{2}^{2}}+\cdots+\frac{\partial^{2}}{\partial x_{n-1}^{2}}\right) \quad(j=1,2 \ldots) .
$$

Recently, Ren and Yang (see [4]) extended Theorems A and B by defining generalised Neumann integrals with continuous functions under less restricted conditions than (1.1). 
Meanwhile, they also proved that for any continuous function $f$ on $\partial \mathcal{T}_{n}$ there exists a solution of Neumann problem on $\mathcal{T}_{n}$. To state them, we need some preliminaries.

Let $L$ and $N$ be two points on $\mathcal{T}_{n}$ and $\partial \mathcal{T}_{n}$, respectively. By $\langle L, N\rangle$ we denote the usual inner product in $\mathbf{R}^{n}$. We denote

$$
|L-N|^{2-n}=\sum_{k=0}^{\infty} d_{k, n}|N|^{-k-n+2}|L|^{k} G_{k, n}(t)
$$

where $|N|>|L|$,

$$
t=|L|^{-1}|N|^{-1}\langle L, N\rangle, \quad d_{k, n}=\left(\begin{array}{c}
k+n-3 \\
k
\end{array}\right)
$$

and $G_{k, n}$ is the $n$-dimensional Legendre polynomial of degree $k$.

As in [2], we shall use the following generalised Dirichlet kernel. For a non-negative integer $l$, two points $L \in \mathcal{T}_{n}$ and $N \in \partial \mathcal{T}_{n}$, we put

$$
\mathbb{V}_{l, n}(L, N)= \begin{cases}-\rho_{n+1} \sum_{k=0}^{l-1} d_{k, n}|N|^{-n-k+2}|L|^{k} G_{k, n}(t) & \text { when }|N| \geq 1 \text { and } l \geq 1 \\ 0 & \text { when }|N|<1 \text { and } l \geq 1 \\ 0 & \text { when } l=0\end{cases}
$$

The generalised Neumann kernel $\mathbb{K}_{l, n}(L, N)$ on $\mathcal{T}_{n}$ is defined by (see [2])

$$
\mathbb{K}_{l, n}(L, N)=\mathbb{K}_{0, n}(L, N)-\mathbb{V}_{l, n}(L, N),
$$

where $L \in \mathcal{T}_{n}, N \in \partial \mathcal{T}_{n}$ and

$$
\mathbb{K}_{0, n}(L, N)=-\alpha_{n}|L-N|^{2-n} .
$$

As for similar generalised Dirichlet kernel in a half plane and smooth cone, we refer the reader to the papers by Yang and Ren (see [5]), Zhao and Yamada (see [6]) and Su (see [1]).

Let $f(N)$ be a continuous function on $\partial \mathcal{T}_{n}$. Then the generalised Neumann integral on $\mathcal{T}_{n}$ can be defined by

$$
\mathbb{H}_{l, n}[f](L)=\int_{\partial \mathcal{T}_{n}} f(N) \mathbb{K}_{l, n}(L, N) d N
$$

Ren and Yang proved the following results.

Theorem C (see [4], Corollary 1) Let $1<p<\infty, n+\beta-2>-(n-1)(p-1)$ and

$$
1-\frac{1-\beta}{p}<m<2-\frac{1-\beta}{p} \text {. }
$$

Let $f(N)\left(N=\left(y^{\prime}, 0\right)\right)$ be a continuous function on $\partial \mathcal{T}_{n}$ such that

$$
\int_{\partial H}\left|f\left(y^{\prime}\right)\right|^{p}\left(1+\left|y^{\prime}\right|\right)^{2-\beta-n} d y^{\prime}<\infty .
$$


Then the generalised Neumann integral $\mathbb{H}_{l, n}[f](L)$ is a solution of the Neumann problem on $\mathcal{T}_{n}$ with respect to $f$ satisfying

$$
\mathrm{M}\left(\left|\mathbb{H}_{l, n}[f]\right|\right)(r)=O\left(|x|^{1+\frac{\beta-1}{p}} \sec ^{n-2} \theta\right)
$$

as $r \rightarrow+\infty$.

Theorem D (see [4], Theorem 3) Let $1 \leq p<\infty, \beta>1-p$, l be a positive integer and

$$
\begin{aligned}
& 1-\frac{1-\beta}{p}<m<2-\frac{1-\beta}{p} \text { when } p>1, \\
& \beta \leq m<\beta+1 \quad \text { when } p=1 .
\end{aligned}
$$

Let $f(N)$ be a continuous function on $\partial \mathcal{T}_{n}$ satisfying (1.3). If $h(L)$ is a solution of the Neumann problem on $\mathcal{T}_{n}$ with respect to $f$ such that

$$
\lim _{r \rightarrow \infty, L=(r, \Theta) \in H} h^{+}(L)=o\left(r^{l+\left[1+\frac{\beta-1}{p}\right]}\right),
$$

then

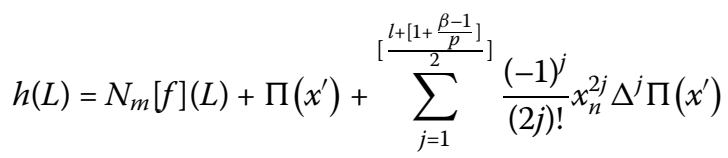

for any $L=\left(x^{\prime}, x_{n}\right)$, where $d$ is a constant, $\Pi\left(x^{\prime}\right)$ is a polynomial of degree less than $l+\left[1+\frac{\beta-1}{p}\right]$ on $\partial \mathcal{T}_{n}$.

From Theorems A, B, C and D, it is easy to see that the continuous boundary function $f$ grows slowly on $\partial \mathcal{T}_{n}$. It is natural to ask what will happen if $f$ is replaced by a fast-growing continuous function on $\partial \mathcal{T}_{n}$. In this paper, we shall solve this problem and explicitly give a new solution of the Neumann problem on $\partial \mathcal{T}_{n}$.

Define

$$
\varepsilon_{0}=\limsup _{r \rightarrow \infty} \tau^{-1}(r) r \tau^{\prime}(r) \log r<1,
$$

where $\tau(r)$ is a nondecreasing and continuously differentiable function satisfying $\tau(r) \geq 1$ for any $r \in \mathbf{R}^{+} \cup\{0\}$.

From these we see that there is a sufficiently large positive number $r$ such that for any $t>r$

$$
\tau(e)(\ln t)^{\epsilon_{0}+\epsilon}>\tau(t)
$$

where $\epsilon$ is a sufficiently small positive number satisfying $\epsilon_{0}+\epsilon<1$.

Let $\mathfrak{A}_{\varpi}$ be the set of continuous functions $f(N)\left(N=\left(y^{\prime}, 0\right)\right)$ on $\partial \mathcal{T}_{n}$ satisfying

$$
\int_{\partial \mathcal{T}_{n}}\left|f\left(y^{\prime}\right)\right|\left(1+\left|y^{\prime}\right|\right)^{3-n-\varpi-\tau\left(\left|y^{\prime}\right|\right)} d y^{\prime}<+\infty,
$$

where $\varpi$ is a real number such that $\varpi>2$. 


\section{Results}

Now we state our results.

Theorem 1 If $\in \mathfrak{A}_{\varpi}$, then generalised Neumann integral $\mathbb{H}_{\left[\tau\left(\left|y^{\prime}\right|\right)+\varpi\right], n}[f](L)$ is a solution of the Neumann problem on $\mathcal{T}_{n}$ with respect to $f$.

Then we shall prove that if the negative part of a harmonic function satisfies a fastgrowing condition, then its positive part satisfies the similar condition. That is to say, the condition of Theorem 1 may be replaced by a weaker integral condition. To state this result, we also need some notations.

Let $\mathfrak{B}_{\varpi}$ be the set of continuous functions $f(N)\left(N=\left(y^{\prime}, y_{n}\right)\right)$ on $\mathcal{T}_{n}$ satisfying

$$
\int_{\mathcal{T}_{n}}|f(N)|(1+|N|)^{1-n-\varpi-\tau(|N|)} y_{n} d N<+\infty
$$

By $\mathfrak{C}_{\varpi}$ we denote the set of all continuous functions $h(N)$ on $\overline{\mathcal{T}_{n}}$, harmonic on $\mathcal{T}_{n}$ with $h^{-}(N) \in \mathfrak{B}_{\varpi}$ and $h^{-}\left(y^{\prime}\right) \in \mathfrak{A}_{\varpi}$.

Theorem 2 The conclusion of Theorem 1 remains valid if its condition is replaced by $h \in$ $\mathfrak{C}_{\varpi}$.

Theorem 3 If $h \in \mathfrak{C}_{\varpi}$, then there exists a harmonic function $\Lambda(L)$ with normal derivative vanishes on $\partial \mathcal{T}_{n}$ such that

$$
h(L)=\Lambda(L)+\mathbb{H}_{\left[\tau\left(\left|y^{\prime}\right|\right)+\varpi\right], n}[h](L),
$$

where $L \in \overline{\mathcal{T}}_{n}$.

\section{Lemmas}

Lemma 1 Let $L \in \mathcal{T}_{n}$ and $N \in \partial \mathcal{T}_{n}$ such that $|N| \geq \max \{1,2|L|\}$. Then (see [7])

$$
\left|\mathbb{K}_{l, n}(L, N)\right| \leq M|N|^{-l-n+2}|L|^{l},
$$

where $M$ is a positive constant.

Lemma 2 Let $\mathbb{W}(L, N)\left(N \in \partial \mathcal{T}_{n}\right)$ be a locally integrable function for any fixed point $L \in \mathcal{T}_{n}$, $g(N)$ be a upper semicontinuous and locally integrable function on $\partial \mathcal{T}_{n}$. Set

$$
\mathbb{K}(L, N)=\mathbb{K}_{0, n}(L, N)-\mathbb{W}(L, N)
$$

for any $N \in \partial \mathcal{T}_{n}$ and $L \in \mathcal{T}_{n}$.

Suppose that the following two conditions hold:

(I) There are a positive number $R$ and a neighborhood $B\left(N^{*}\right)$ of $N^{*}\left(\in \partial \mathcal{T}_{n}\right)$ satisfying

$$
\int_{\partial \mathcal{T}_{n}[R,+\infty) \cup \partial \mathcal{T}_{n}(-\infty,-R]}|g(N)|\left|\frac{\partial}{\partial x_{n}} \mathbb{K}(L, N)\right| d N<\epsilon
$$

where $\epsilon>0$. 
(II) There exists a positive number $R$ satisfying

$$
\limsup _{L \rightarrow N^{*}, L \in \mathcal{T}_{n}} \int_{\partial \mathcal{T}_{n}(-R, R)}|g(N)|\left|\frac{\partial}{\partial x_{n}} \mathbb{W}(L, N)\right| d N=0
$$

for any $N^{*} \in \partial \mathcal{T}_{n}$.

Then

$$
\limsup _{L \rightarrow N^{*} \in \partial \mathcal{T}_{n}, L \in \mathcal{T}_{n}} \int_{\partial \mathcal{T}_{n}} g(N) \frac{\partial}{\partial x_{n}} \mathbb{W}(L, N) d N \leq g\left(N^{*}\right) .
$$

Proof Let $N^{*}$ be any point of $\partial \mathcal{T}_{n}$ and $\epsilon$ be any positive number. There exists a positive number $R^{*}$ satisfying

$$
\int_{\partial \mathcal{T}_{n}\left[R^{*},+\infty\right) \cup \partial \mathcal{T}_{n}\left(-\infty,-R^{*}\right]}|g(N)|\left|\frac{\partial}{\partial x_{n}} \mathbb{K}(L, N)\right| d N \leq \frac{\epsilon}{2}
$$

for any $L=\left(x^{\prime}, x_{n}\right) \in \mathcal{T}_{n} \cap B\left(N^{*}\right)$ from $(\mathrm{I})$.

Let $\phi$ be a continuous function on $\partial \mathcal{T}_{n}$ such that $0 \leq \phi \leq 1$ and

$$
\phi= \begin{cases}1 & \text { if } \partial \mathcal{T}_{n}\left[-R^{*}, R^{*}\right] \\ 0 & \text { if } \partial \mathcal{T}_{n}\left(-\infty,-2 R^{*}\right) \cup \partial \mathcal{T}_{n}\left(2 R^{*},+\infty\right)\end{cases}
$$

Let $\mathbb{K}_{0, n}^{j}(L, N)$ be the Neumann function of $\mathcal{T}_{n}(-j, j)$, where $j$ is a positive integer. Since

$$
\Gamma_{j}(L, N)=\mathbb{K}_{0, n}(L, N)-\mathbb{K}_{0, n}^{j}(L, N)
$$

on $\mathcal{T}_{n}(-j, j)$ converges monotonically to 0 as $j \rightarrow \infty$, we can find an integer $j^{*}$ satisfying $j^{*}>2 R^{*}$ such that

$$
\int_{\partial \mathcal{T}_{n}\left(-2 R^{*}, 2 R^{*}\right)}|\phi(N) g(N)|\left|\frac{\partial}{\partial x_{n}} \Gamma_{j^{*}}(L, N)\right| d \sigma<\frac{\epsilon}{4}
$$

for any $L=\left(x^{\prime}, x_{n}\right) \in B\left(N^{*}\right) \cap \mathcal{T}_{n}$.

Then we have from (3.2) and (3.3) that

$$
\begin{aligned}
\int_{\partial \mathcal{T}_{n}} g(N) \frac{\partial}{\partial x_{n}} \mathbb{K}(L, N) d N \leq & \int_{\partial \mathcal{T}_{n}\left(-2 R^{*}, 2 R^{*}\right)} g(N) \frac{\partial \mathbb{K}_{0, n}^{j^{*}}(L, N)}{\partial x_{n}} \phi(N) d N \\
& +\int_{\partial \mathcal{T}_{n}\left(-2 R^{*}, 2 R^{*}\right)}|g(N)| \frac{\partial \Gamma_{j^{*}}(L, N)}{\partial x_{n}}|| \phi(N) \mid d N \\
& +\int_{\partial \mathcal{T}_{n}\left(-2 R^{*}, 2 R^{*}\right)}|g(N)| \frac{\partial \mathbb{W}(L, N)}{\partial x_{n}} \mid d N \\
& +2 \int_{\partial \mathcal{T}_{n}\left[R^{*},+\infty\right) \cup \partial \mathcal{T}_{n}\left(-\infty,-R^{*}\right]}|g(N)|\left|\frac{\partial \mathbb{K}(L, N)}{\partial x_{n}}\right| d N \\
\leq & \int_{S_{n}\left(\Gamma ;\left(-2 R^{*}, 2 R^{*}\right)\right)} g(N) \frac{\partial \mathbb{K}_{0, n}^{j^{*}}(L, N)}{\partial x_{n}} \phi(N) d N \\
& +\int_{\partial \mathcal{T}_{n}\left(-2 R^{*}, 2 R^{*}\right)}|g(N)| \frac{\partial \mathbb{W}(L, N)}{\partial x_{n}} \mid d N+\frac{5}{4} \epsilon
\end{aligned}
$$

for any $L=\left(x^{\prime}, x_{n}\right) \in \mathcal{T}_{n} \cap B\left(N^{*}\right)$. 
Consider an upper semicontinuous function

$$
\psi(N)= \begin{cases}\phi(N) g(N) & \text { if } \partial \mathcal{T}_{n}\left[-2 R^{*}, 2 R^{*}\right], \\ 0 & \text { if } \partial \mathcal{T}_{n}\left[-j^{*}, j^{*}\right]-\partial \mathcal{T}_{n}\left[-2 R^{*}, 2 R^{*}\right]\end{cases}
$$

on $\partial \mathcal{T}_{n}\left(-j^{*}, j^{*}\right)$ and denote the Perron-Wiener-Brelot solution of the Neumann problem on $\mathcal{T}_{n}\left(-j^{*}, j^{*}\right)$ by $\mathbb{H}_{\psi}\left(L ; \mathcal{T}_{n}\left(-j^{*}, j^{*}\right)\right)$. We know that

$$
\int_{\partial \mathcal{T}_{n}\left(-2 R^{*}, 2 R^{*}\right)} g(N) \frac{\partial \mathbb{K}_{0, n}^{j^{*}}(L, N)}{\partial x_{n}} \phi(N) d N=\mathbb{H}_{\psi}\left(L ; \mathcal{T}_{n}\left(-j^{*}, j^{*}\right)\right) .
$$

We also have

$$
\limsup _{L \rightarrow N^{*}, L \in \mathcal{T}_{n}} \mathbb{H}_{\psi}\left(L ; \mathcal{T}_{n}\left(-j^{*}, j^{*}\right)\right) \leq \limsup _{N \in \partial T_{n}, N \rightarrow N^{*}} \psi(N)=g\left(N^{*}\right)
$$

Hence we obtain

$$
\limsup _{L \rightarrow N^{*}, L \in \mathcal{T}_{n}} \int_{\partial \mathcal{T}_{n}\left(-2 R^{*}, 2 R^{*}\right)} g(N) \frac{\partial \mathbb{K}_{0, n}^{j^{*}}(L, N)}{\partial x_{n}} \phi(N) d N \leq g\left(N^{*}\right),
$$

which together with (II) and (3.4) gives (3.1).

Lemma 3 Let $r>1$ and $h(N)\left(N=\left(y^{\prime}, y_{n}\right)\right)$ be a function harmonic on $\mathcal{T}_{n}$. Then

$$
\int_{S_{n}(r)} r^{-1-n} h(N) n y_{n} d N+\int_{\partial \mathcal{T}_{n}(1, r)} h\left(y^{\prime}\right)\left(\left|y^{\prime}\right|^{-n}-r^{-n}\right) d y^{\prime}=d_{1}+d_{2} r^{-n}
$$

where

$$
d_{1}=\int_{S_{n}(1)} y_{n}\left((n-1) h(N)+\frac{\partial h(N)}{\partial n}\right) d N
$$

and

$$
d_{2}=\int_{S_{n}(1)} y_{n}\left(h(N)-\frac{\partial h(N)}{\partial n}\right) d N .
$$

\section{Proof of Theorem 1}

We have from (1.4)

$$
M_{1}(r) \geq(2 r)^{\tau(k+1)+\varpi+1} k^{\frac{2-\varpi}{2}}
$$

for any $k>k_{r}=[2 r]+1$, where $M_{1}(r)$ is a positive constant dependent only on $r$.

We have for any $L \in \mathcal{T}_{n}$ and $|L| \leq R$

$$
\begin{aligned}
& \sum_{k=k_{r}}^{\infty} \int_{\partial \mathcal{T}_{n}[k, k+1)}\left|f\left(y^{\prime}\right)\right|(2|L|)^{\left[\tau\left(\left|y^{\prime}\right|\right)+\varpi\right]}\left|y^{\prime}\right|^{2-n-\left[\tau\left(\left|y^{\prime}\right|\right)+\varpi\right]} d y^{\prime} \\
& \quad \leq \sum_{k=k_{r}}^{\infty} k^{\frac{2-\sigma}{2}}(2 r)^{1+\infty+\tau(k+1)} \int_{\partial \mathcal{T}_{n}[k, k+1)} 2\left|f\left(y^{\prime}\right)\right|\left(1+\left|y^{\prime}\right|\right)^{1-n-\frac{\omega-2}{2}-\tau\left(\left|y^{\prime}\right|\right)} d y^{\prime}
\end{aligned}
$$




$$
\begin{aligned}
& \leq 2 M_{1}(r) \int_{\partial \mathcal{T}_{n}\left[k_{r},+\infty\right)}\left|f\left(y^{\prime}\right)\right|\left(1+\left|y^{\prime}\right|\right)^{1-n-\frac{\omega-2}{2}-\tau\left(\left|y^{\prime}\right|\right)} d y^{\prime} \\
& <+\infty
\end{aligned}
$$

from Lemma 1 and (1.5). So $\mathbb{H}_{\left[\tau\left(\left|y^{\prime}\right|\right)+\varpi\right], n}(L)$ is absolutely convergent.

Next we shall prove that

$$
\lim _{L \rightarrow N^{\prime}, L=\left(x^{\prime}, x_{n}\right) \in \mathcal{T}_{n}} \frac{\partial \mathbb{H}_{\left[\tau\left(\left|y^{\prime}\right|\right)+\varpi\right], n}(L)}{\partial x_{n}}=h\left(N^{\prime}\right)
$$

for any $N^{\prime}=\left(y^{\prime}, 0\right) \in \partial \mathcal{T}_{n}$. By applying Lemma 2 to $-g\left(y^{\prime}\right)$ and $g\left(y^{\prime}\right)$ by setting

$$
\mathbb{W}(L, N)=\mathbb{V}_{\left[\tau\left(\left|y^{\prime}\right|\right)+\varpi\right], n}(L, N),
$$

then we shall see that (I) and (II) hold. Take any $N^{\prime}=\left(y^{\prime}, 0\right) \in \partial \mathcal{T}_{n}$ and any $\epsilon>0$. There exists a number $R\left(>\max \left\{2\left(\delta+y^{\prime}\right), 1\right\}\right)$ satisfying

$$
\int_{\partial \mathcal{T}_{n}[R,+\infty) \cup \partial \mathcal{T}_{n}(-\infty,-R]}|f(N)|\left|\frac{\partial}{\partial x_{n}} \mathbb{K}_{\left[\tau\left(\left|y^{\prime}\right|\right)+\varpi\right], n}(L, N)\right| d N<\epsilon
$$

for any $L \in \mathcal{T}_{n} \cap U\left(N^{\prime}, \delta\right)$ from (1.5) and (4.2), which is (I) in Lemma 2. To see (II), we only need to observe from (1.2) that for any $N^{\prime} \in \partial \mathcal{T}_{n}$

$$
\limsup _{L=\left(x^{\prime}, x_{n}\right) \rightarrow N^{*}, L \in \mathcal{T}_{n}} \frac{\partial}{\partial x_{n}} \mathbb{V}_{\left[\tau\left(\left|y^{\prime}\right|\right)+\varpi\right], n}(L, N)=0 .
$$

So Theorem 1 is proved.

\section{Proof of Theorem 2}

Lemma 2 gives

$$
\begin{aligned}
P_{-}(r)+\int_{\partial \mathcal{T}_{n}(1, r)} h^{-}\left(y^{\prime}\right)\left(\left|y^{\prime}\right|^{-n}-r^{-n}\right) d y^{\prime} \\
=P_{+}(r)+\int_{\partial \mathcal{T}_{n}(1, r)} h^{+}\left(y^{\prime}\right)\left(\left|y^{\prime}\right|^{-n}-r^{-n}\right) d y^{\prime}-d_{1}-d_{2} r^{-n},
\end{aligned}
$$

where

$$
P_{ \pm}(r)=\int_{\sigma_{n}(r)} n h^{ \pm}(y) r^{-n-1} y_{n} d N
$$

Since $h \in \mathfrak{C}_{\varpi}$, we obtain by $(2.1)$

$$
\int_{1}^{+\infty} P_{-}(r) r^{2-\varpi-\tau(r)} d r=n \int_{\mathcal{T}_{n}(1,+\infty)} h^{-}(N) y_{n}|N|^{1-\varpi-n-\tau(|N|)} d N<+\infty .
$$

We have by (1.5)

$$
\begin{aligned}
& \int_{1}^{+\infty} r^{2-\varpi-\tau(r)}\left(\int_{\partial \mathcal{T}_{n}(1, r)} h^{-}\left(y^{\prime}\right)\left(\left|y^{\prime}\right|^{-n}-r^{-n}\right) d y^{\prime}\right) d r \\
& =\int_{\partial \mathcal{T}_{n}(1,+\infty)} h^{-}\left(y^{\prime}\right)\left(\int_{\left|y^{\prime}\right|}^{\infty} r^{2-\varpi-\tau(r)}\left(\left|y^{\prime}\right|^{-n}-r^{-n}\right) d r\right) d y^{\prime}
\end{aligned}
$$




$$
\begin{aligned}
& \leq \frac{n}{n+1} \int_{\partial \mathcal{T}_{n}(1,+\infty)} h^{-}\left(y^{\prime}\right)\left|y^{\prime}\right|^{3-\varpi-n-\tau\left(\left|y^{\prime}\right|\right)} d y^{\prime} \\
& <+\infty
\end{aligned}
$$

From (5.1), (5.2) and Lemma 2, we see that

$$
\begin{aligned}
& \int_{1}^{+\infty} r^{\frac{2-\varpi}{2}-\tau(r)}\left(\int_{\partial \mathcal{T}_{n}(1, r)} h^{+}\left(y^{\prime}\right)\left(\left|y^{\prime}\right|^{-n}-r^{-n}\right) d y^{\prime}\right) d r \\
& =\int_{\partial \mathcal{T}_{n}[1,+\infty)} h^{+}\left(y^{\prime}\right)\left(\int_{\left|y^{\prime}\right|}^{\infty} r^{\frac{2-\varpi}{2}-\tau(r)}\left(\left|y^{\prime}\right|^{-n}-r^{-n}\right) d r\right) d y^{\prime} \\
& \leq \int_{1}^{+\infty} P_{-}(r) r^{\frac{2-\varpi}{2}-\tau(r)} d r-\int_{1}^{+\infty} r^{\frac{2-\varpi}{2}-\tau(r)}\left(d_{1}+d_{2} r^{-n}\right) d r \\
& \quad+\int_{1}^{+\infty} r^{\frac{2-\varpi}{2}-\tau(r)}\left(\int_{\partial \mathcal{T}_{n}(1, r)} h^{-}\left(y^{\prime}\right)\left(\left|y^{\prime}\right|^{-n}-r^{-n}\right) d y^{\prime}\right) d r \\
& <+\infty
\end{aligned}
$$

Set

$$
\mathbb{Q}(\varpi)=\lim _{\left|y^{\prime}\right| \rightarrow \infty} \int_{\left|y^{\prime}\right|}^{\infty} r^{\frac{2-\varpi}{2}-\tau(r)}\left(\left|y^{\prime}\right|^{-n}-r^{-n}\right) d r\left|y^{\prime}\right|^{-3+\varpi+n+\tau\left(\left|y^{\prime}\right|\right)} .
$$

It is easy to see that

$$
\mathbb{Q}(\varpi)=+\infty
$$

from (1.4), which shows that

$$
M_{2}\left|y^{\prime}\right|^{3-\varpi-n-\tau\left(\left|y^{\prime}\right|\right)} \leq \int_{\left|y^{\prime}\right|}^{\infty} r^{\frac{2-\varpi}{2}-\tau(r)}\left(\left|y^{\prime}\right|^{-n}-r^{-n}\right) d r
$$

for any $\left|y^{\prime}\right| \geq 1$, where $M_{2}$ is a positive constant.

It follows that

$$
\begin{aligned}
& M_{2} \int_{\partial \mathcal{T}_{n}[1,+\infty)} h^{+}\left(y^{\prime}\right)\left|y^{\prime}\right|^{3-\varpi-n-\tau\left(\left|y^{\prime}\right|\right)} d x^{\prime} \\
& \quad \leq \int_{\partial \mathcal{T}_{n}[1,+\infty)} h^{+}\left(y^{\prime}\right) \int_{\left|y^{\prime}\right|}^{\infty} r^{\frac{2-\varpi}{2}-\tau(r)}\left(\left|y^{\prime}\right|^{-n}-r^{-n}\right) d r d y^{\prime} \\
& \quad<+\infty
\end{aligned}
$$

from (5.3).

Then Theorem 2 is proved from $|h|=h^{+}+h^{-}$.

\section{Proof of Theorem 3}

Put $h^{\prime}(L)=h(L)-\mathbb{H}_{\left[\tau\left(\left|y^{\prime}\right|\right)+\varpi\right], n}(L)$. Then it is easy to see that $h^{\prime}(L)$ is harmonic on $\mathcal{T}_{n}$ with normal derivative vanishes on $\partial \mathcal{T}_{n}$ and $h^{\prime}(L)$ can be continuously extended to $\overline{\mathcal{T}_{n}}$. By applying the Schwarz reflection principle [8], p.68, to $h^{\prime}(L)$, it follows that there is a function harmonic on $\mathcal{T}_{n}$ satisfying $h\left(L^{*}\right)=-h^{\prime}(L)=-\left(h(L)-\mathbb{H}_{\left[\tau\left(\left|y^{\prime}\right|\right)+\varpi\right], n}(L)\right)$ for $L \in \bar{T}_{n}$, where * 
denotes reflection in $\partial \mathcal{T}_{n}$ just as $L^{*}=\left(x^{\prime},-x_{n}\right)$. Thus $h(L)=\Lambda(L)+\mathbb{H}_{\left[\tau\left(\left|y^{\prime}\right|\right)+\varpi\right], n}(L)$ for all $L \in \overline{\mathcal{T}}_{n}$, where $\Lambda(L)$ is a harmonic function on $\mathcal{T}_{n}$ with normal derivative which vanishes continuously on $\partial \mathcal{T}_{n}$. Theorem 3 is proved.

\section{Competing interests}

The authors declare that they have no competing interests.

\section{Authors' contributions}

All authors contributed equally and significantly in writing this article. All authors read and approved the final manuscript.

\section{Author details}

${ }^{1}$ School of Science, Zhejiang University of Science and Technology, Hangzhou, 310023, China. ${ }^{2}$ Department of Mathematics and Computer Science, Faculty of Science, Alexandria University, Alexandria, 21511, Egypt.

\section{Acknowledgements}

This work was supported by the Natural Science Foundation of Zhejiang Province (No. LQ13A010019). The authors would like to thank the referee for invaluable comments and insightful suggestions.

Received: 2 August 2016 Accepted: 21 September 2016 Published online: 12 October 2016

\section{References}

1. Su, B: Dirichlet problem for the Schrödinger operator in a half space. Abstr. Appl. Anal. 2012, Article ID 578197 (2012)

2. Su, B: Growth properties of harmonic functions in the upper half-space. Acta Math. Sin. 55(6), 1095-1100 (2012)

3. Armitage, D: The Neumann problem for a function harmonic in $R^{n} \times(0, \infty)$. Arch. Ration. Mech. Anal. 63(1), 89-105 (1976)

4. Ren, Y, Yang, P: Growth estimates for modified Neumann integrals in a half space. J. Inequal. Appl. 2013, Article ID 572 (2013)

5. Yang, D, Ren, Y: Dirichlet problem on the upper half space. Proc. Indian Acad. Sci. Math. Sci. 124(2), 175-178 (2014)

6. Zhao, T, Yamada, A Jr.: A remark on the Dirichlet problem in a half-plane. J. Inequal. Appl. 2014, Article ID 497 (2014)

7. Hayman, W, Kennedy, P: Subharmonic Functions. Vol. I. London Mathematical Society Monographs, vol. 9. Academic Press, London (1976)

8. Axler, S, Bourdon, P, Ramey, W: Harmonic Function Theory, 2nd edn. Springer, New York (1992)

\section{Submit your manuscript to a SpringerOpen ${ }^{\circ}$ journal and benefit from:}

- Convenient online submission

Rigorous peer review

- Immediate publication on acceptance

- Open access: articles freely available online

- High visibility within the field

- Retaining the copyright to your article 